\title{
Caveolin-1 Genetic Polymorphisms Interact with Fatty Acid Types to Modulate Metabolic Syndrome Risk
}

\author{
Faezeh Abaj $^{1}$ and khadiheh MIRZAE ${ }^{1}$ \\ ${ }^{1}$ Tehran University of Medical Sciences
}

November 30, 2020

\begin{abstract}
Background: Metabolic syndrome (MetS) is related with all-cause mortality. Caveolin-1 (Cav-1) has been widely studied in dyslipidemia, and several studies have indicated that Cav-1 genetic variations may correlate with dietary intake of fatty acids. The aim of the current study was therefore to evaluate the interaction of Cav-1 rs3807992 with types of dietary fatty acid in MetS risk factor status Methods: This cross-sectional study was carried out on 404 overweight and obese females. Dietary intake was obtained from a 147-item FFQ. The CAV-1 genotype was measured using the PCR-RFLP method. Anthropometric values and serum levels (TC, LDL, HDL, TG, FBS) were measured by standard methods. Results: It was observed that the $(\mathrm{AA}+\mathrm{AG})$ group had significantly higher $\mathrm{BMI}, \mathrm{WC}$ and $\mathrm{DBP}(\mathrm{P}=0.02, \mathrm{P}=0.02$ and $\mathrm{P}=0.01$, respectively) and lower serum LDL, HDL and TC $(\mathrm{P}<0.05)$ than the GG group. It was found that A allele carriers were at higher odds of MetS $(\mathrm{P}=0.01)$, abdominal obesity $(\mathrm{P}=0.06)$, increased $\mathrm{TG}$ concentration $(\mathrm{P}=0.01)$, elevated blood pressure $(\mathrm{BP})(\mathrm{P}=0.01)$, increased glucose concentration $(\mathrm{P}=0.45)$, and decreased HDL-cholesterol concentration $(\mathrm{P}=0.03)$. Moreover, the interaction of Cav-1 and $\mathrm{SFA}$ intake was significant in terms of $\operatorname{MetS}(\mathrm{P}=0.01)$, LDL $(\mathrm{P}=0.03)$, DBP $(\mathrm{P}=0.01)$ and LDL/HDL $(\mathrm{P}=0.05)$. Additionally, the $(\mathrm{AA}+\mathrm{AG})$ group was significantly related to $\mathrm{PUFA}$ intake in terms of MetS $(\mathrm{P}=0.04)$, TG $(\mathrm{P}=0.02)$, glucose $(\mathrm{P}=0.02)$ and HOMA-IR $(\mathrm{P}=0.01)$. Conclusions: Higher PUFA consumption might attenuate the Cav-1 rs3807992 associations with MetS, and individuals with greater genetic predisposition appeared to have a higher risk of MetS, associated with higher SFA consumption To date, studies on this polymorphism have been animal studies and have not been performed on healthy and obese human society For the first time, this study provides information on the interaction of different fatty acids with the Caveolin gene, which is functionally effective in lipid metabolism
\end{abstract}

\section{Introduction}

Metabolic syndrome (MetS) is related with metabolic abnormalities including obesity, hyperglycemia, dyslipidemia, and hypertension, and is prevalent worldwide ${ }^{1}$. MetS is related to all-cause mortality, myocardial infarction (MI), and stroke in patients with or without diabetes ${ }^{2}$. Surprisingly, the evidence demonstrates that genetic variations play a main part in the prevention and treatment of various chronic disease, particularly in MetS $^{3-5}$. MetS and cardiovascular diseases (CVD) also demonstrate the role of genetic and environmental factors in diet-related disorders. A genetic background of metabolic disorders has been shown to relate to death rate over the last few decades ${ }^{6}$. There is therefore a need to identify the genes that derive MetS and to develop new therapies. Caveolin-1 (Cav-1) is a key protein component of caveolae, and has been widely studied in dyslipidemia and CVD due to signal transduction, trafficking in cholesterol hemostasis, and triacylglycerol metabolism ${ }^{7}$. Mice treated with Cav-1 are resistant to high-fat diets, and they show lipodystrophy, hypertension, insulin resistance, and abnormal glucose metabolism ${ }^{8}$. Moreover, several studies have indicated that Cav-1 genetic variations might interact with other risk factors, including dietary intake of fatty acids, suggesting a positive association between Cav-1 and hypercholesterolemia ${ }^{9}$. In studies conducted among Caucasian and Hispanic cohorts, the prevalence of Cav-1 gene variant rs926198 is related with higher odds of MetS risk and low HDL 10,11. Also, Cav-1 overexpression has been observed to relate to higher odds 
of atherosclerosis in experimental models ${ }^{12}$. The exact mechanisms are unclear, but it seems that Cav-1 is able to regulate several key enzymes in lipid metabolism, such as cholesterol ester transfer protein and phospholipid transfer protein ${ }^{13}$. While the association between Cav-1 polymorphisms and type 2 diabetes risk has been widely reported in various populations ${ }^{14}$, these relationships with MetS have been inconsistent, despite several publications on the association between Cav- 1 gene variants and serum lipid profiles ${ }^{14-17}$. To the authors' knowledge, there has been no study evaluating Cav-1 rs3807992 variant, metabolic risk factor, and the interaction of fatty acid intake levels with this SNP. Hence, the aim of the current study was to evaluate the interaction of Cav-1 genetic polymorphism with the types of dietary fatty acids, in terms of MetS risk factor status.

\section{Material and Methods}

\section{Study Population}

A total of 404 women in the range of 18-55 years old were randomly selected from among participants in a cross-sectional study performed in 2019.Participants provided written informed consent. The inclusion criteria were: obese or overweight, no alcohol consumption, and no smoking. The exclusion criteria were: CVDs, kidney failure, stroke, thyroid disease, liver disease, cancer, inflammatory illnesses, and those taking any therapeutic medications. Each participant was interviewed in order to obtain demographic data, then referred to the laboratory for blood sampling. Anthropometric measures were taken, including: height $(\mathrm{m})$, weight $(\mathrm{kg})$, waist circumference $(\mathrm{WC}, \mathrm{cm})$ measured at the narrowest part of the abdomen, and body mass index (BMI, $\mathrm{kg} / \mathrm{m}^{2}$, calculated by dividing weight by height squared). Blood pressure (BP) was measured with a sphygmomanometer (BP) after 5 minutes' rest. Triglyceride (TG) (mg/dl), highdensity lipoprotein (HDL) (mg/dl), total cholesterol (mg/dl), and low-density lipoprotein (LDL) (mg/dl) were measured according to standard protocols. The study was approved by the Ethics Committee at the Tehran University of Medical Sciences (TUMS) (97-03-161-41017).

\section{MetS Definition}

MetS cases were required to meet three or more of the following criteria according to the Adult Treatment Panel III (ATP III) criteria:

1) Elevated fasting blood glucose FPG[?]100 mg/dl.

2) Hypertriglyceridemia TG[?]150 mg/dl

3) Elevated blood pressure ([?]130/85 mmHg)

4) Low (HDL-c) $<50 \mathrm{mg} / \mathrm{dl}$ in women

5) WC cut-off $80 \mathrm{~cm}$ [women] was considered as an indicator of abdominal obesity ${ }^{18}$.

\section{Genotyping}

For genotyping the Cav-1 polymorphisms, DNA was extracted from whole blood via a Mini Columns kit (Type G; Genall; Exgene). The polymerase chain reaction-restriction fragment length polymorphism (PCRRFLP) technique was employed to investigate Cav-1 polymorphisms (rs3807992) in gene fragments (major allele $\mathrm{G}$ and minor allele A). PCR was carried out using the following primers: F :3'AGTATTGACCTGATTTGCCATG5'R :5'GTCTTCTGGAAAAAGCACATGA-3'. PCR reactions were performed in a volume of $20 \mu \mathrm{l}$, containing $1 \mu \mathrm{l}$ extracted DNA, $1 \mu \mathrm{l}$ Forward primers, $1 \mu \mathrm{l}$ Reverse primers, $7 \mu \mathrm{l}$ distilled water and $10 \mu \mathrm{l}$ Taq DNA Polymerase Master Mix in a DNA thermocycler. The DNA templates were denatured at $94{ }^{\circ} \mathrm{C}$ for $3 \mathrm{~min}$ and 40 cycles, including a min denaturation at $94{ }^{\circ} \mathrm{C}$, a min annealing at $42-50{ }^{\circ} \mathrm{C}$ and elongation at $72{ }^{\circ} \mathrm{C}$ for $2 \mathrm{~min}$. Amplified DNA was digested with Hin1II (NIaIII) restriction enzyme at $37^{\circ} \mathrm{C}$ overnight, then separated by electrophoresis on an agarose gel (2\%). Fragments concluding three genotypes of the Cav-1 rs3807992 variant were detected: uncut homozygous AA (213bp), cut heterozygous GA (3 bands: $118 \& 95 \& 213 \mathrm{bp}$ ) and cut homozygous GG (2 bands: $118 \& 95 \mathrm{bp})$.

\section{Statistical Analysis}


Statistical analysis was performed using SPSS v.25 software (SPSS Inc., IL, USA) and significance level was considered $\mathrm{P}<0.05$. The Kolmogorov-Smirnov was utilized to test the normality of the data, and all data were expressed as means $\pm \mathrm{SD}$. The Pearson's chi-square test was used to determine the Hardy-Weinberg Equilibrium deviation among allele frequency of Cav-1 G32124A (rs3807992). Independent-sample t-test was used to evaluate the differences between the two groups, and ANCOVA test was used to evaluate for confounding effects. Binary logistic regression was used to estimate interactions between rs3807992 and high and low dietary fat intake on the Odds Ratio (OR) of the MetS component. All ORs were adjusted for variables proven to be related to MetS components, (such as age, educational level, smoking status, physical activity and energy intake).

\section{Dietary Assessment}

The Food Frequency Questionnaire (FFQ) was a useful tool for evaluating dietary intake, and included 147 items ${ }^{19}$. This assessment was carried out by interviewing the occurrence of food items consumed on the basis of a predetermined list of foods. The extracted FFQ values were then changed to grams/day. For the evaluation of macro- and micronutrient content, N4 software was used; all measurements were then entered into IBM SPPS.

\section{Results}

\section{Clinical Characteristics According to Cav-1 rs3807992 Genotypes}

As shown in Table 1, 404 women with a mean age of 36.67 years were evaluated in the present study. Table 1 expresses the mean clinical characteristics of all women according to Cav- 1 genotypes. It was observed that the ( $\mathrm{AA}+\mathrm{AG}$ ) group had significantly higher $\mathrm{BMI}$, WC and $\mathrm{DBP}(\mathrm{P}=0.02, \mathrm{P}=0.02$ and $\mathrm{P}=0.01$ respectively). After adjustment for confounders (age, energy intake, physical activity and DBP), BMI and DBP remained significant. Furthermore, women with $(\mathrm{AA}+\mathrm{AG})$ allele had significantly lower serum LDL, HDL and TC (P $<0.05)$ than the GG group. Additionally, no significant differences were detected for age, height, weight, FBG, TG and SBP.

\section{Genotype Frequencies between MetS and Control Groups}

Distributions of allele frequencies and the effect size of rs3807992 on various genetic models (codominant, dominant, recessive) had significant differences between MetS and control groups. In the codominant model, the A homozygous allele for the Cav-1 (rs3807992) gene was $52 \%$ higher in the control group than the MetS group (OR $2.52[1.11,5.70], P=0.02$ ). In the dominant model, the A homozygous allele for the Cav-1 (rs3807992) gene was 31\% higher in the control group than the MetS group (OR 2.31[1.16,4.61], $P=0.01$ ) (Table 2).

\section{Associations between Cav-1 rs3807992 and the Risk of MetS and Its Components}

It was found that (rs3807992) A allele carriers were at higher odds of MetS (OR 2.31 [1.16, 4.16], $P$ $=0.01$ ), abdominal obesity (OR 1.42[0.98,2.06], $P=0.06$ ), increased TG concentration (OR 2.12 [1.13,3.95], $P=0.01)$, elevated blood pressure (BP) (OR 7.03 [1.43,34.44], $P=0.01$ ), increased glucose concentration (OR 0.7[0.27,1.78], $P=0.45)$, and decreased HDL-cholesterol concentration (OR -1.4 [1.02,1.93] $P=0.03)$ (Table 3).

\section{Dietary Intake Relationships between Cav-1 rs3807992 Gene Polymorphisms, and their Inter- action on MetS and Its Components}

The results of dietary nutritional intake are described according genotype groups, which show that the $(\mathrm{AA}+\mathrm{AG})$ group had increased SFA and cholesterol consumption (g/d), compared to the GG group. No significant differences were found in other nutrient consumption values (Table 4). It was observed that the interaction of Cav-1 rs3807992 gene polymorphism and SFA intake was significant on MetS (OR 2.31 $[1.16,4.61], P=0.01$ ) and its components, including LDL (OR 12.95 [1.21,24.69], $P=0.03$ ), DBP (OR 6.52 $[1.59,11.45], P=0.01)$ and LDL/HDL (OR $0.28[-0.01,0.57], P=0.05)$ in a crude model. These values remained significant in the multi-adjusted model (adjusted for age, energy intake, BMI, smoking status, age at onset 
of obesity, and total PUFA intake). There was no further significant interaction between the (AA+AG) group and SFA intake on other biochemical parameters, including HDL, TG, TC and LDL/HDL, in both the crude and adjusted models $(\mathrm{P}>0.05)$ (Table 5). Interestingly, when Cav-1 (AA+AG) group interaction was analyzed by PUFA intake, the relationship was significant in terms of MetS (OR -0.207 [0.04, 0.95], $P=$ $0.04)$ and its components, including TG (OR $-0.27[0.05,0.8], P=0.02)$, glucose (OR -0.06 [0.005, 0.75], $P=$ $0.02)$ and HOMA-IR (OR $-0.22[0.06,0.78], P=0.01)$ in the crude model. These remained significant in the adjusted model (adjusted for age, energy intake, BMI, smoking status, age at onset of obesity) (Table 6).

\section{Discussion}

According to these findings, female A-allele carriers had significantly higher BMI, WC and DBP, and had lower serum LDL, HDL and TC, compared to GG genotypes. This demonstrates that their clinical parameters are predisposed to MetS. A-allele carriers were at higher odds of MetS. To the authors' knowledge, there is little evidence describing an association between Cav-1 and metabolic syndrome. It was observed that a genetic variant of Cav-1 (rs3807992) was associated with increased MetS risk, which is consistent with previous studies that revealed a significant association between the minor allele in cav-1 variations and the odds of metabolic diseases ${ }^{10,11,15,17}$. Also, compared with other candidate gene studies, no studies had evaluated SNP rs3807992 and MetS risk. It is proposed that Cav-1 polymorphisms increase MetS risk through altered Cav-1 gene expression, attenuating dyslipidemia and hypertension, while impairing glucose and insulin homeostasis ${ }^{12-14,20}$. Cav-1 regulates signaling molecules, such as IRS1, that have a key role in appropriate insulin responses, PKA, angiotensin II receptors, active blood pressure molecules, and binding sites for calcium ions; all of these may affect various clinical traits of MetS ${ }^{14,21,22}$. Additionally, Cav-1 is able to effect NO, insulin, lipids, and hormone metabolisms ${ }^{23}$. Therefore, caveolae and its components may become useful sites for further investigation into treating MetS.

The genetic association between Cav-1 polymorphisms and MetS by dietary fat intake was reported. High dietary SFA intake ([?]25gr) especially accentuated the negative effects of rs3807992 in terms of MetS risk. Furthermore, a potential gene-nutrient interaction was found between the rs3807992 Cav-1 polymorphism and PUFA intake. High PUFA intake ([?]6\% energy) reduced the negative effects of rs3807992 in terms of MetS risk, with the greatest protection achieved by A-allele carriers. To the authors' knowledge, this interaction has not been previously studied, and the present paper's finding support the necessity of lower and higher consumption of SFA and PUFA, respectively, in the diets of A-allele carriers. Most previous intervention studies have shown that a higher intake of SFA was detrimental to maintaining insulin sensitivity, whereas PUFA showed beneficial effects ${ }^{24}$. Additionally, environmental factors, in particular dietary composition, may alter the risk of MetS. Nutrigenetic research has indicated that dietary fat background can influence genotype-phenotype relations ${ }^{25}$.

In line with previous studies, experimental studies have reported that sphingomyelin is a key phospholipid of caveolae. SFA intake may lead to increases in sphingolipids levels in the cardiac cell membranes, thus disrupting the caveolae contents ${ }^{26,27}$. Moreover, Chapkin et al. reported that in animal models, n-3 PUFA intake may modulate the function of caveolae proteins/lipid, affecting membrane fusion and cell-cell signaling, and improving insulin signaling. Many studies have indicated that H-Ras and eNOS (endothelial nitric oxide synthase) are moved from caveolae in n-3 PUFA supplemented rats, which suppressed the Ras-dependent signaling and reduced blood pressure, thereby lowering the MetS risk ${ }^{28}$. Caveolae membrane fatty acids (in the internal and external leaflet) is also significantly altered by n-3 PUFA intake, and is even able to change the function of the caveolae.

Caveolae plays a unique function in the uptake of various lipid and glucose metabolites. Accordingly, caveolae is a main center for several nutrient metabolisms through the cell membrane ${ }^{29}$. Caveolae is able to uptake fatty acid, triacylglycerol, and cholesterol in many tissues, which leads to an elevation in caveolae density in obese rats ${ }^{30,31}$. However, in these animals, the number of caveolae decreases in the arterial endothelium and at the ends of smooth muscle cells, leading to dyslipidemia ${ }^{32}$.

The mechanism observed in the present study, by which fatty acids are able to modify the genetic risk 
posed by Cav-1 polymorphisms, remains unknown; further studies are needed to indicate such gene-diet interventions. Several limitations can also be identified in the current study. Dietary intake was assessed by a food-frequency questionnaire (FFQ), which is self-reported and thus dependent on patient memory. Due to financial limitations, it was not possible to perform western blot analysis to determine whether rs-3807992 SNP alters the expression of Cav-1. The focus of the current study was on dietary fat composition, but other nutrient components, including carbohydrates or fiber, can also play a role in the progression of MetS. Finally, given the observational nature of the study, it is not possible to tell whether the associations which were identified in women (but not men) are of a causal nature.

\section{Conclusion}

To the authors' knowledge, this is the first study presenting the association of a genetic variant of Cav-1 rs3807992 with the risk of MetS and its components, including TG, BP, and HDL level. However, further studies are needed to determine the strength of this association in a larger population; the contribution of this study is the novel finding that rs3807992 clearly predicts MetS among obese women. Analyses of the individual components of MetS confirmed that the rs3807992 variant is related to elevated BP, dyslipidemia, low HDL cholesterol, and high TG levels. Also, Cav-1 rs3807992 genotypes are sensitive to dietary SFA and PUFA, which allows individuals to monitor and adjust SFA and PUFA consumption accordingly. Finally, these results can be used in combination with a patient's genetic history in order to provide more applicable and tailored nutritional advice for preventing or attenuating MetS in overweight and obese women.

\section{Acknowledgments :}

We would like to thank all the individuals who participated in this project. This study was supported by a grant from Tehran University of Medical Sciences (97-03-161-41017)

\section{Conflict of Interest :}

The authors declare no conflict of interest

\section{List of Abbreviations:}

BMI: Body mass index

CVD: Cardiovascular disease DBP: Diastolic blood pressure DHA: Docosahexaenoic acid EPA: Eicosapentaenoic acid FBS:

FFQ: Food frequency questionnaire

FA: Fatty acid

GWAS: Genome-wide association studies

LDL: Low-density lipoprotein MA: Minor Allele MetS: Metabolic syndrome

PCR: Polymerase chain reaction

RFLP: Restriction fragment length polymorphism

SNP: Single nucleotide polymorphism

TC: Total cholesterol

TG: Triglyceride

WC: Waist circumference

\section{References}

1. Beltrán-Sánchez H, Harhay MO, Harhay MM, McElligott SJJotACoC. Prevalence and trends of metabolic syndrome in the adult US population, 1999-2010. 2013;62(8):697-703.

2. Zhuo Q, Yang W, Chen J, Wang YJNRR. Metabolic syndrome meets osteoarthritis. 2012;8(12):729.

3. Katzmarzyk PT, Pérusse L, Rice T, Rao DC, Bouchard C. Familial aggregation of seven-year changes in blood pressure in Canada. The Canadian journal of cardiology. 2001;17(12):1267-1274.

4. Monda KL, North KE, Hunt SC, Rao DC, Province MA, Kraja AT. The genetics of obesity and the 
metabolic syndrome. Endocrine, metabolic $\mathscr{E}$ immune disorders drug targets. 2010;10(2):86-108.

5. Pérusse L, Després JP, Tremblay A, et al. Genetic and environmental determinants of serum lipids and lipoproteins in French Canadian families. Arteriosclerosis (Dallas, Tex). 1989;9(3):308-318.

6. Wang H, Dong S, Xu H, Qian J, Yang JJMbr. Genetic variants in FTO associated with metabolic syndrome: a meta-and gene-based analysis. 2012;39(5):5691-5698.

7. Jin Y, Lee S-J, Minshall RD, Choi AMJAJoP-LC, Physiology M. Caveolin-1: a critical regulator of lung injury. 2011;300(2):L151-L160.

8. Pavlides S, Gutierrez-Pajares JL, Danilo C, Lisanti MP, Frank PG. Atherosclerosis, caveolae and caveolin1. In: Caveolins and Caveolae. Springer; 2012:127-144.

9. Shyu HY, Chen MH, Hsieh YH, et al. Association of eNOS and Cav-1 gene polymorphisms with susceptibility risk of large artery atherosclerotic stroke. PloS one. 2017;12(3):e0174110.

10. Baudranda R. A prevalent caveolin-1 gene variant is associated with the metabolic syndrome in Caucasians and Hispanics. 2015.

11. Pojoga LH, Underwood PC, Goodarzi MO, et al. Variants of the caveolin-1 gene: a translational investigation linking insulin resistance and hypertension. J Clin Endocrinol Metab.2011;96(8):E1288-1292.

12. Fernandez-Hernando C, Yu J, Davalos A, Prendergast J, Sessa WC. Endothelial-specific overexpression of caveolin-1 accelerates atherosclerosis in apolipoprotein E-deficient mice. The American journal of pathology. 2010;177(2):998-1003.

13. Frank PG, Pavlides S, Cheung MW, Daumer K, Lisanti MP. Role of caveolin-1 in the regulation of lipoprotein metabolism. American journal of physiology Cell physiology. 2008;295(1):C242-248.

14. Haddad D, Al Madhoun A, Nizam R, Al-Mulla F. Role of Caveolin-1 in Diabetes and Its Complications. Oxidative Medicine and Cellular Longevity. 2020;2020:9761539.

15. Chen S, Wang X, Wang J, et al. Genomic variant in CAV1 increases susceptibility to coronary artery disease and myocardial infarction.Atherosclerosis. 2016;246:148-156.

16. Mora-Garcia G, Gomez-Camargo D, Alario A, Gomez-Alegria C. A Common Variation in the Caveolin 1 Gene Is Associated with High Serum Triglycerides and Metabolic Syndrome in an Admixed Latin American Population. Metab Syndr Relat Disord. 2018;16(9):453-463.

17. Nizam R, Al-Ozairi E, Goodson JM, et al. Caveolin-1 Variant Is Associated With the Metabolic Syndrome in Kuwaiti Children. Front Genet. 2018;9:689.

18. Hu FB, Rimm EB, Stampfer MJ, Ascherio A, Spiegelman D, Willett WC. Prospective study of major dietary patterns and risk of coronary heart disease in men. The American journal of clinical nutrition.2000;72(4):912-921.

19. Mohammadifard N, Sajjadi F, Maghroun M, Alikhasi H, Nilforoushzadeh F, Sarrafzadegan N. Validation of a simplified food frequency questionnaire for the assessment of dietary habits in Iranian adults: Isfahan Healthy Heart Program, Iran. ARYA atherosclerosis.2015;11(2):139-146.

20. Razani B, Woodman SE, Lisanti MP. Caveolae: from cell biology to animal physiology. Pharmacological reviews. 2002;54(3):431-467.

21. Czikora I, Feher A, Lucas R, Fulton DJ, Bagi Z. Caveolin-1 prevents sustained angiotensin II-induced resistance artery constriction and obesity-induced high blood pressure. Am J Physiol Heart Circ Physiol. 2015;308(5):H376-385.

22. Daniel EE, Eteraf T, Sommer B, Cho WJ, Elyazbi A. The role of caveolae and caveolin 1 in calcium handling in pacing and contraction of mouse intestine. J Cell Mol Med. 2009;13(2):352-364. 
23. Wang H, Wang AX, Liu Z, Chai W, Barrett EJ. The trafficking/interaction of eNOS and caveolin-1 induced by insulin modulates endothelial nitric oxide production. Molecular endocrinology (Baltimore, Md). 2009;23(10):1613-1623.

24. Iggman D, Risérus U. Role of different dietary saturated fatty acids for cardiometabolic risk. Clinical Lipidology. 2011;6(2):209-223.

25. Phillips CM. Nutrigenetics and metabolic disease: current status and implications for personalised nutrition. Nutrients.2013;5(1):32-57.

26. Le Lay S, Li Q, Proschogo N, et al. Caveolin-1-dependent and -independent membrane domains. Journal of lipid research.2009;50(8):1609-1620.

27. Perona JS. Membrane lipid alterations in the metabolic syndrome and the role of dietary oils. Biochim Biophys Acta Biomembr.2017;1859(9 Pt B):1690-1703.

28. Chapkin RS, McMurray DN, Davidson LA, Patil BS, Fan YY, Lupton JR. Bioactive dietary long-chain fatty acids: emerging mechanisms of action. The British journal of nutrition. 2008;100(6):1152-1157.

29. Pilch PF, Liu L. Fat caves: caveolae, lipid trafficking and lipid metabolism in adipocytes. Trends Endocrinol Metab.2011;22(8):318-324.

30. Grayson TH, Chadha PS, Bertrand PP, et al. Increased caveolae density and caveolin-1 expression accompany impaired NO-mediated vasorelaxation in diet-induced obesity. Histochemistry and cell biology. 2013;139(2):309-321.

31. Su X, Abumrad NA. Cellular fatty acid uptake: a pathway under construction. Trends Endocrinol Metab. $2009 ; 20(2): 72-77$.

32. Howitt L, Grayson TH, Morris MJ, Sandow SL, Murphy TV. Dietary obesity increases NO and inhibits BKCa-mediated, endothelium-dependent dilation in rat cremaster muscle artery: association with caveolins and caveolae. Am J Physiol Heart Circ Physiol.2012;302(12):H2464-2476.

\section{Hosted file}

Tables.pdf available at https://authorea.com/users/380085/articles/496204-caveolin-1genetic-polymorphisms-interact-with-fatty-acid-types-to-modulate-metabolic-syndrome-risk

\section{Hosted file}

Figures.pdf available at https://authorea.com/users/380085/articles/496204-caveolin-1genetic-polymorphisms-interact-with-fatty-acid-types-to-modulate-metabolic-syndrome-risk 